\title{
Genome-wide association study of carcass weight in commercial Hanwoo cattle
}

\author{
Zewdu Edea', Yeong Ho Jeoung ${ }^{2}$, Sung-Sub Shin ${ }^{3}$, Jaeul Ku${ }^{4}$, Sungbo Seo ${ }^{4}$, Il-Hoi Kim ${ }^{1}$, \\ Sang-Wook Kim ${ }^{1}$, and Kwan-Suk Kim ${ }^{1, *}$
}

\author{
* Corresponding Author: Kwan-Suk Kim \\ Tel: +82-43-261-2547, Fax: +82-43-273-2240, \\ E-mail: kwanskim@chungbuk.ac.kr \\ 1 Department of Animal Science, Chungbuk National \\ University, Cheongju 28644, Korea \\ ${ }^{2}$ Korea Animal Improvement Association, Seoul \\ 06668, Korea \\ ${ }^{3}$ Korea Institute for Animal products Quality \\ Evaluation, Sejong 30100, Korea \\ ${ }^{4}$ Biomedical Research Center, Turbosoft Inc. Cheongju \\ 28161, Korea \\ ORCID \\ Kwan-Suk Kim \\ https://orcid.org/0000-0002-5895-4398
}

Submitted Apr 11, 2017; Revised Jul 12, 2017; Accepted Oct 22, 2017
Objective: The objective of the present study was to validate genes and genomic regions associated with carcass weight using a low-density single nucleotide polymorphism (SNP) Chip in Hanwoo cattle breed.

Methods: Commercial Hanwoo steers $(\mathrm{n}=220)$ were genotyped with $20 \mathrm{~K}$ GeneSeek genomic profiler BeadChip. After applying the quality control of criteria of a call rate $\geq 90 \%$ and minor allele frequency (MAF) $\geq 0.01$, a total of 15,235 autosomal SNPs were left for genomewide association (GWA) analysis. The GWA tests were performed using single-locus mixed linear model. Age at slaughter was fitted as fixed effect and sire included as a covariate. The level of genome-wide significance was set at $3.28 \times 10^{-6}(0.05 / 15,235)$, corresponding to Bonferroni correction for 15,235 multiple independent tests.

Results: By employing EMMAX approach which is based on a mixed linear model and accounts for population stratification and relatedness, we identified 17 and 16 loci significantly $(\mathrm{p}<0.001)$ associated with carcass weight for the additive and dominant models, respectively. The second most significant ( $\mathrm{p}=0.000049)$ SNP (ARS-BFGL-NGS-28234) on bovine chromosome 4 (BTA4) at $21 \mathrm{Mb}$ had an allele substitution effect of $43.45 \mathrm{~kg}$. Some of the identified regions on BTA2, 6, 14, 22, and 24 were previously reported to be associated with quantitative trait loci for carcass weight in several beef cattle breeds.

Conclusion: This is the first genome-wide association study using SNP chips on commercial Hanwoo steers, and some of the loci newly identified in this study may help to better DNA markers that determine increased beef production in commercial Hanwoo cattle. Further studies using a larger sample size will allow confirmation of the candidates identified in this study.

Keywords: Carcass Weight; Genome-Wide Association Studies (GWAS); Hanwoo Cattle; Quantitative Trait Loci (QTL)

\section{INTRODUCTION}

The Korean native cattle have inhabited in the Korean Peninsula for over 2000 years. Mitochondrial based analysis showed that Korea cattle carried only Bos taurus haplotype [1]. Hanwoo cattle breed has been phenotypically subjected to intensive selection for meat production and quality traits including carcass weight, eye muscle area, backfat thickness, and marbling score [2]. As a result, a substantial genetic gain has achieved for carcass weight and eye muscle area [3]. Similarly, a yearling weight was increased from $315.54 \mathrm{~kg}$ to $355.06 \mathrm{~kg}$ over the last 13 years [3].

Carcass weight is one of the most critical quantitative traits affecting beef cattle production. And it needs to be taken into consideration in genetic improvement programs to assess the economic return on investment in beef cattle production. Carcass weight is consider- 
ably affected by genetics and environmental factors [4]. Bos taurus and Bos indicus $\times$ Bos taurus breeds had greater mean live weight, carcass weight and dressing percentage than pure Bos indicus breed [5]. Similarly, within Bos taurus breeds, late maturing breeds produces a heavier carcass with little fat, while early maturing breeds produce lighter weight carcasses [4].

With the advent of genome sequencing and genotyping platforms, genome-wide association studies (GWAS) have become a powerful tool and increasingly used to dissect the genetics of quantitative trait loci (QTL) in several domestic livestock species. In cattle, different genotyping platforms including the Illumina Bovine 50K (San Diego, CA, USA), 770K and 10 chips (Santa Clara, CA, USA) have been developed during the last few years [6]. GWAS correlate allele frequencies at the entire genome level and provides a high resolution to map loci even with modest effects on QTLs [7,8].

With the availability of such genotyping arrays, GWAS have been performed to dissect the genetics of QTL in several beef cattle breeds $[9,10]$. Particularly, GWAS based studies have uncovered several genomic regions that harbor QTLs for carcass weight in various cattle breeds. For instance, Lee et al [11] genotyped 1,011 Hanwoo steers obtained from progeny testing programs using a high-density single nucleotide polymorphism (SNP) chip and detected QTL for carcass weight on bovine chromosome 14 (BTA14, 23 to $25 \mathrm{Mb}$ ). Using a low- density SNP chip [12] reported QTL for carcass weight on BTA4 in Korean Hanwoo cattle. In Japanese Black cattle [13], mapped markers associated with carcass weight on chromosomes 6, 8 , and 14 . Another study by [14] detected significant linkages with carcass weight on BTA 1, 6, 7, and 10. Recently, Sorbolini et al [15] used high-density assay and identified significant SNPs on BTA2 associated with carcass weight and average daily gain in Marchiana cattle breed. Additionally, GWAS revealed the association of SNPs within diacylglycerol O-acyl transferase 1 and leptin receptor gene with beef traits [16]. Nonsynonymous SNP on BTA 6 within non-SMC condensin I complex subunit $\mathrm{G}$ identified to be significantly associated with carcass weight in a large Japanese black population [10].

Although over the past few years, GWAS have identified several QTLs in Hanwoo steers of progeny tested populations, to date no GWAS have been conducted on carcass weight of commercial Hanwoo steers. Though there is little information on QTLs for carcass weight at different growth stages, studies in inbred mice found that QTLs affecting early and late growth traits were distinct and mapped to different chromosomes, indicatingthat different genes and physiological mechanisms may control body growth at different stages [17]. Similarly, age specific QTLs for growth rate were reported in crossbred chickens [18]. In human, it has been demonstrated that age significantly affected the expression of genes related to metabolism and cell development in skeletal muscle [19]. Therefore, different loci may control body weight/carcass weight at differ- ent ages in cattle. In the present study, we performed a GWAS using 220 commercial Hanwoo steers genotyped on 20KGGP (GeneSeek genomic profiler) array (Gene Seek Inc., Lincoln, NE, USA) to identify loci that are significantly associated with carcass weight. The results of the current study will aid the development of selection tool in commercial Hanwoo cattle populations.

\section{MATERIALS AND METHODS}

\section{Animals, genotyping, and quality control}

Nasal samples were collected using Performagene LIVESTOCK's nasal swab DNA collection kit (DNA Genotek Inc., Kanata, ON, Canada) from 220 Hanwoo steers from commercial Hanwoo production farms. The 220 animals were sired by 30 bulls ranging from 1 to 38 calves per sire. Carcass weight was measured at an average age of 31.07 months with an average value of $442.45 \pm 45.98 \mathrm{~kg}$. All animals were genotyped on GeneSeek genomic profiler BeadChip (Gene Seek Inc., USA) according to the manufacture's protocol (http://www.illumina. com) that contains 19,720 SNPs of which, 18,593 were mapped to autosomes, 1,023 to X-chromosome, 9 to $\mathrm{Y}$-chromosome, 13 to $\mathrm{mtDNA}$, and 82 not assigned to any chromosome. SNPs on sex chromosomes, mtDNA and not assigned to any genomic location were removed from further analyses. Quality control was performed with SNP and Variation Suit v8.4.4 (Golden Helix, Inc., Bozeman, MT, USA www.goldenhelix. com). Four individuals with missing genotype greater than $10 \%$ were excluded. After applying quality control by individuals, a total of 18,593 autosomal SNPs with an average spacing of $135.10 \mathrm{~kb}$ were filtered for a call rate $\geq 90 \%$ and minor allele frequency (MAF) $\geq 0.01$. We also filtered out SNPs that significantly deviated from Hardy-Weinberg equilibrium (HEW) with $\mathrm{p}<1 \times 10^{-6}$. Subsequently, 3,223 SNPs that had MAF less than $0.01,89$ SNPs departed from the HWE and 121 SNPs with a call rate less than $90 \%$ (10\% missing) were excluded from analysis. After applying the above quality control criteria, a total of 15,235 autosomal SNPs left for 220 Hanwoo steers were used for genome-wide association analysis.

\section{Statistical analyses}

Genome-wide association (GWA) tests were performed using single-locus mixed linear model GWAS (EMMAX) using SNP and Variation Suit v8.5.0 (Golden Helix, Inc., USA www. goldenhelix.com). In GWAS, lack of accounting for population structure may lead to spurious association results [20]. The EMMAX (Efficient Mixed-Model Association expedited) statistic test approach is proven to outperform the principal analysis and genomic control in accounting for sample structure (stratification and relatedness) [20]. In our analysis, this technique was used to correct for confounding effects due to subpopulation structure and relatedness between individuals. 
In the EMMAX, the $n \times n$ genetic matrix of identity by state between individuals is computed from the genotype data. The model used can be expressed as:

$$
\mathrm{y}=X \boldsymbol{\beta}+\mathrm{Zu}+\boldsymbol{e}
$$

Where $y$ is an $n \times 1$ the vector of observed phenotypic values of the animals, $\boldsymbol{X}$ is an $\boldsymbol{n} \times \boldsymbol{f}$ matrix of fixed effects including mean SNPs and age (month), $\beta$ is a $q \times 1$ vector representing coefficients of the fixed effects, $Z$ is an $n \times t$ relating the instances of the random effect to the carcass weight, $\beta$ coefficient of fixed effects, $\boldsymbol{u}$ the random animal effects, and $\boldsymbol{e}$ residual effect. The proportion of variance explained ( $p v e$ ) by the effects of the marker was estimated with SNP and Variation Suit v8.5.0 (Golden Helix, Inc., USA www.goldenhelix.com) using the EMMA technique [20]. Age at slaughter was fitted as fixed effect and sire is included as a covariate. We tested the additive and dominance genotypic models. The level of genome-wide significance was set at $3.28 \times 10^{-6}(0.05 / 15,235)$, corresponding to Bonferroni correction for 15,235 multiple independent tests. The general linear model (GLM) procedure of the Statistical Analysis (V.9.1, SAS Inc., Cary, NC, USA) was used to test for significant difference between the genotypes of the 17 SNPs that are significantly associated $(\mathrm{p}<0.001)$ with carcass weight. We further used a haplotype-based approach to identify variants associated with carcass weight. Haplotype blocks were defined according to Gabriel method [21]. According to this method, SNP pairs to be in strong linkage disequilibrium (LD) if the one-sided upper $95 \%$ confidence bound on $D^{\prime}$ is 0.98 (that is consistent with no historical recombination) and the lower bound is above 0.7. Haplotype trend regression analysis was performed using SNP and Variation Suit v8.4.4 (Golden Helix, Inc., USA www.goldenhelix.com). Genes within the significant SNPs and their close vicinity were explored from National Center for Biotechnology Information database (http://www.ncbi.nlm.nih.gov/). We have further searched for the QTL regions overlap with the identified candidate regions from bovine QTL database (http://bovinegenome.org/bovineqtl_v2/findQTL.html) and (http://www.animalgenome. org/cgi-bin/QTLdb/BT/index). Gene network analysis provides an important insight into the genetic architecture of complex polygenic traits [22]. To this end, we carried out gene network analysis using the candidate genes by employing GeneMANIA (www.genemania.org/plugin/) implemented in Cytoscape software 3.4 [23].

\section{RESULTS AND DISCUSSION}

In the current study, we collected carcass weight from and genotyped 220 Hanwoo steers with GeneSeek genomic profiler BeadChip that contains 19,720 SNPs. Of these, 15,235 autosomal SNPs that met the quality control criteria were used for the genome-wide association analysis. In GWAS, significant SNPs were declared when the $\mathrm{p}$ value was less than the genome-wide type I error rate, adjusted with Bonferroni correction by using $\alpha / K$, where $\alpha=0.05$ and $K=$ number of SNPs. After applying a Bonferroni correction $(0.05 / 15,235=3.28$ $\times 10^{-6}$ ), however, we did not detect any SNP displaying the set genome-wide significant threshold for the models tested (Figure 1, Table 1) but this was not unexpected given the limited number of samples used in our study $(\mathrm{n}=220)$. In addition, as indicated in the previous studies, the lack of significance at the genome-wide level because this is a stringent test [24]. A total of 17 and 16 SNPs were found to be associated with carcass weight across the bovine genome at the $0.1 \%$ significant level for the additive and dominant models, respectively (Tables 1, 2).

For the additive model, the 17 significant SNPs $(\mathrm{p}<0.001)$ were distributed over 15 BTA containing 6 annotated genes, including LOC101903200, poly (ADP-ribose) polymerase family member 4 (PARP4), glycoprotein A33 (GPA33), NAD kinase (NADK), phosphatidylinositol-3,4,5-trisphosphate dependent Rac exchange factor 2 (PREX2), and FERM domain containing $4 \mathrm{~B}$ (FRMD4B). The most significant association was observed with Hapmap60145-rs29013637 ( $\mathrm{p}=0.000027$ ) on BTA 24 at $27.38 \mathrm{Mb}$. This SNP is an intergenic and $124 \mathrm{~kb}$ away from the TRNAS-GGA [transfer RNA serine (anticodon GGA)] gene. The second most significant SNP (ARS-BFGLNGS-28234; $\mathrm{p}=0.000049$ ) was located on BTA 14 at $21 \mathrm{Mb}$ (Table 1, Figure 1).

Allele substitution effects for the significant SNPs ranged from 16.47 (BovineHD0900007660) to $43.45 \mathrm{~kg}$ (ARS-BFGLNGS-28234). The most significant SNP (Hapmap60145-rs

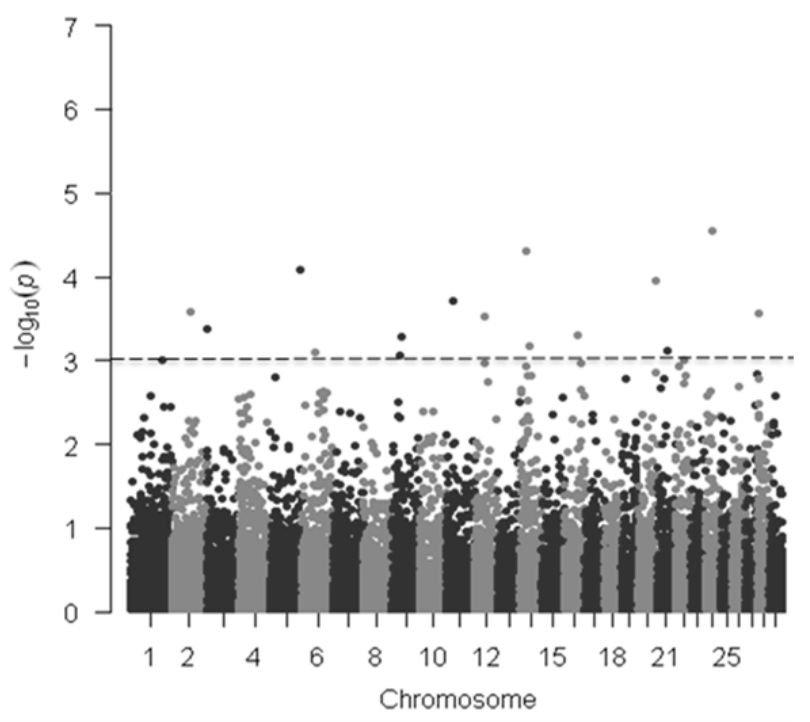

Figure 1. Manhattan plots of genome-wide -log10 (p-values) for carcass weight in Hanwoo cattle for additive model. The dotted horizontal line represents the set significant threshold $(-\log 10$ [p-value $=3]$ ). 
Table 1. Summary of significant SNPs ( $p<0.001)$ associated with carcass weight in 220 Hanwoo cattle using the additive model

\begin{tabular}{|c|c|c|c|c|c|c|c|c|c|c|c|}
\hline SNP & $\mathrm{Chr}$ & Position & $-\log 10$ (p-value) & Gene $^{1)}$ & Reported carcass QTL & $\beta(\mathrm{kg})$ & $V_{g}$ & MAF & $\mathrm{d}_{0}$ & $\mathrm{~d}_{1}$ & $d^{2}$ \\
\hline Hapmap60145-rs29013637 & 24 & 27381101 & 4.55 & - & $26.6-30.0 \mathrm{Mb}$ & -22.57 & 0.090 & 0.43 & 41 & 105 & 74 \\
\hline ARS-BFGL-NGS-28234 & 14 & 21343649 & 4.30 & - & 21.1-21.1 Mb & 43.45 & 0.084 & 0.07 & 1 & 30 & 189 \\
\hline ARS-BFGL-NGS-7347 & 20 & 67090770 & 3.95 & - & - & 20.28 & 0.077 & 0.50 & 54 & 112 & 54 \\
\hline BovineHD1100008236 & 11 & 27645835 & 3.71 & - & - & -25.14 & 0.072 & 0.28 & 18 & 88 & 112 \\
\hline BovineHD0200020296 & 2 & 70647552 & 3.58 & - & 62.2-75.6 Mb & -32.19 & 0.069 & 0.08 & 0 & 37 & 183 \\
\hline ARS-BFGL-NGS-22647 & 3 & 1779182 & 3.37 & GPA33 & - & -34.34 & 0.064 & 0.07 & 2 & 26 & 191 \\
\hline BTB-00646159 & 16 & 52211744 & 3.30 & NADK & $50.0-51.0 \mathrm{Mb}$ & 18.63 & 0.063 & 0.43 & 37 & 117 & 66 \\
\hline BovineHD0900009902 & 9 & 36090095 & 3.28 & - & - & 19.25 & 0.063 & 0.46 & 41 & 119 & 60 \\
\hline ARS-BFGL-NGS-6936 & 14 & 34148235 & 3.17 & PREX2 & 28.1-46.8 Mb & 30.88 & 0.060 & 0.10 & 1 & 42 & 177 \\
\hline BTB-02072074 & 21 & 38226290 & 3.12 & - & - & 22.19 & 0.059 & 0.39 & 28 & 116 & 75 \\
\hline BovineHD0100034972 & 1 & 123851563 & 3.01 & - & 26.6-30.0 Mb & -22.76 & 0.057 & 0.18 & 7 & 66 & 146 \\
\hline
\end{tabular}

SNP, single nucleotide polymorphism; QTL, quantitative trait loci; $\beta$, the allele substitution effect; $V_{g}$, the proportion of variance explained by each SNP; MAF, minor allele frequency; $d_{0}, d_{1}, d_{2}$, the number of individuals with 2,1 , and copies of the minor allele.

1) PARP4, poly (ADP-ribose) polymerase family member 4; GPA33, glycoprotein A33; NADK, NAD kinase; PREX2, phosphatidylinositol-3,4,5-trisphosphate dependent Rac exchange factor 2; FRMD4B, FERM domain containing $4 \mathrm{~B}$.

29013637) had an allele substitution effect of $22.57 \mathrm{~kg}$, whereas the second most significant SNP (ARS-BFGL-NGS-28234) had an effect of $43.45 \mathrm{~kg}$ (Table 1). It was notable that PREX2 and GPA33 genes were significant for both additive and dominant models.

To gain additional information on the loci that had a large effect on observed phenotypic variance, we further calculated the proportion of genetic variance explained by each SNP finding that the most significant SNP on BTA24 at $27.38 \mathrm{Mb}$ explained $0.09 \%$ of the total variance, whereas the second most significant SNP accounted for about $0.08 \%$ of the total variance (Table 1). The proportion of variance explained by

Table 2. Summary of significant SNPs $(p<0.001)$ associated with carcass weight in 220 Hanwoo cattle using the dominant model

\begin{tabular}{lcccccccccccc}
\hline Marker & Chr & Position & Gene $^{1)}$ & Reported carcass QTL & -log10 (p-value) & $\boldsymbol{\beta}(\mathbf{k g})$ & $\mathbf{V}_{\mathbf{g}}$ & $\mathbf{M A F}^{\mathbf{d}_{0}}$ & $\mathbf{d}^{1}$ & $\mathbf{d}_{\mathbf{2}}$ \\
\hline ARS-BFGL-NGS-28234 & 14 & 21343649 & - & $21.9-29.3 \mathrm{Mb}$ & 4.86 & 48.51 & 0.096 & 0.07 & 1 & 30 & 189 \\
BTB-02072074 & 21 & 38226290 & - & - & 4.02 & 33.88 & 0.078 & 0.39 & 28 & 116 & 75 \\
BovineHD2600007390 & 26 & 27840203 & SORCS1 & - & 3.83 & 25.69 & 0.074 & 0.28 & 16 & 89 & 115 \\
ARS-BFGL-NGS-22647 & 3 & 1779182 & GPA33 & - & 3.78 & -40.34 & 0.073 & 0.07 & 2 & 26 & 191 \\
BovineHD0200020296 & 2 & 70647552 & - & $62.2-75.6 \mathrm{Mb}$ & 3.58 & -32.19 & 0.069 & 0.08 & 0 & 37 & 183 \\
ARS-BFGL-NGS-7347 & 20 & 67090770 & - & - & 3.51 & 29.63 & 0.067 & 0.5 & 54 & 112 & 54 \\
ARS-BFGL-NGS-6936 & 14 & 34148235 & PREX2 & $28.1-46.8 \mathrm{Mb}$ & 3.49 & 33.68 & 0.067 & 0.1 & 1 & 42 & 177 \\
BovineHD0600019726 & 6 & 70908736 & - & $53.1-72.2 \mathrm{Mb}$ & 3.48 & 28.81 & 0.067 & 0.45 & 40 & 119 & 61 \\
BovineHD2000011491 & 20 & 40180680 & ADAMTS12 & - & 3.45 & 29.05 & 0.066 & 0.41 & 38 & 104 & 78 \\
ARS-BFGL-NGS-110665 & 22 & 33423142 & FAM19A1 & $28.5-45.7 \mathrm{Mb}$ & 3.42 & 26.03 & 0.065 & 0.42 & 35 & 113 & 72 \\
BTA-26576-n0-rS & 16 & 67703949 & SWT1 & - & 3.36 & 29.07 & 0.064 & 0.17 & 8 & 59 & 153 \\
ARS-BFGL-NGS-111513 & 1 & 125725305 & - & - & 3.27 & 26.03 & 0.062 & 0.49 & 52 & 103 & 56 \\
BovineHD0400002851 & 4 & 9215747 & AKAP9 & $0.8-22.4 \mathrm{Mb}$ & 3.22 & 25.16 & 0.061 & 0.29 & 15 & 95 & 109 \\
Hapmap29357-BTA-147904 & 17 & 32009576 & - & $30.1-32.2 \mathrm{Mb}$ & 3.17 & -25.99 & 0.06 & 0.27 & 25 & 68 & 127 \\
BTB-00874898 & 28 & 6575192 & - & $4.3-8.7 \mathrm{Mb}$ & 3.15 & -32.21 & 0.06 & 0.09 & 2 & 37 & 181 \\
BovineHD2200017653 & 22 & 60652435 & - & - & 3.01 & -26.29 & 0.057 & 0.48 & 53 & 103 & 64 \\
\hline
\end{tabular}

SNP, single nucleotide polymorphism; QTL, quantitative trait loci; $\beta$, the allele substitution effect; $V_{g}$, the proportion of variance explained by each SNP; MAF, minor allele frequency; $d_{0}, d_{1}, d_{2}$, the number of individuals with 2, 1 and copies of the minor allele.

1) SORCS1, sortilin related VPS10 domain containing receptor 1; GPA33, glycoprotein A33; PREX2, phosphatidylinositol-3,4,5-trisphosphate dependent Rac exchange factor 2; ADAMTS12, ADAM metallopeptidase with thrombospondin type 1 motif 12; FAM19A1, family with sequence similarity 19 member A1; SWT1, RNA endoribonuclease homolog; AKAP9, A-kinase anchoring protein 9. 
each significant SNP was very low showing that the polygenic nature of carcass weight is governed by many genes with small effects. In our GLM analysis of the effect of genotype on carcass weight, 4 SNPs (ARS-BFGL-NGS-21717, BovineHD090000 7660, BovineHD0900009902, and BTB-00646159) had significant effect on carcass weight $(\mathrm{p}<0.05)$ (Figure 2).

Eight of the 17 SNP coincide with carcass weight QTLs in various beef cattle breeds. The regions on BTA14 at $21 \mathrm{Mb}$ and $34 \mathrm{Mb}$ were reported to harbor QTL for carcass weights in beef cattle breeds. A number of previous studies identified QTL for carcass weight, bovine stature, and growth related traits in cattle on bovine BTA14. In Hanwoo cattle, Lee et al [25] identified SNPs significantly association with carcass weight on BTA 14 (23 Mb to $25 \mathrm{Mb}$ ). In addition, [9] identified QTL for carcass weight on BTA 14 harboring pleomorphic adenoma gene 1 (PLAG1) gene in Japanese Black cattle. In Nellore cattle, GWAS using 777K SNPs [26] also detected association for carcass weight on BTA14. Moreover, Rempel et al [27] identified carcass weight QTL (21.1 to $21.1 \mathrm{Mb}$ ) on BTA14 in crossbred beef cattle. It has been also reported that the QTLs on BTA14 associated with body size in taurine and zebu cattle
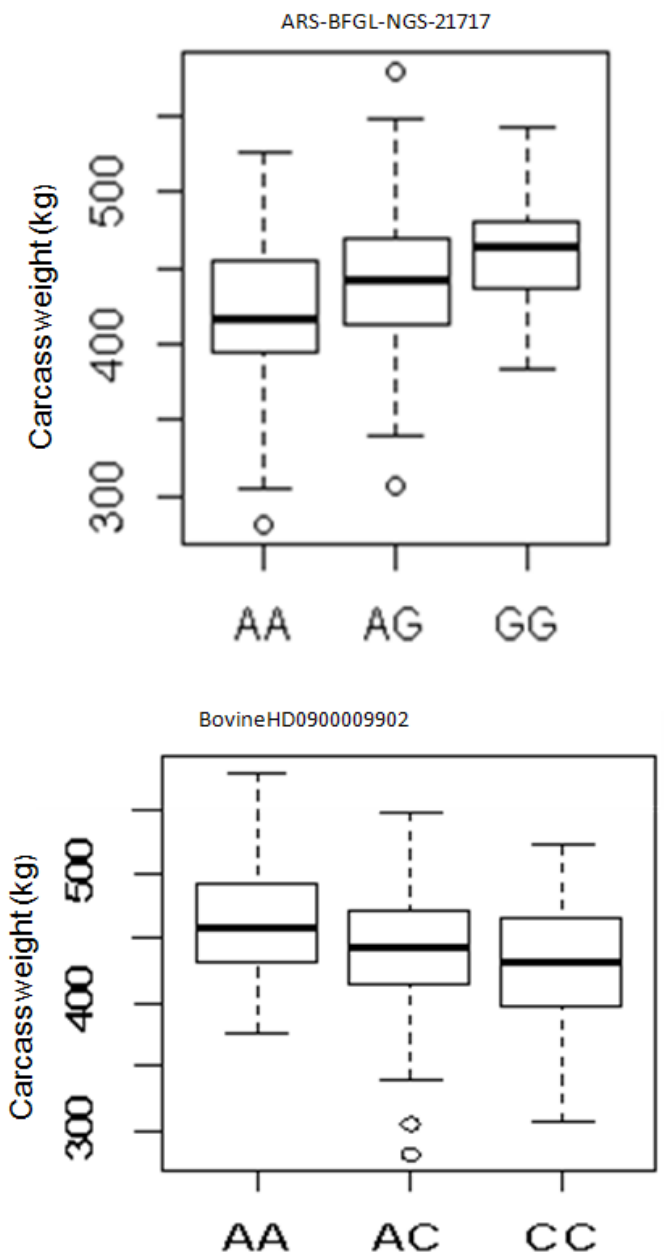

[28].

The other SNP (BTB-00874898) that was significantly associated with carcass weight $(\mathrm{p}=0.0002)$ was located at 6.5 $\mathrm{Mb}$ of chromosome 28 . On the same chromosome (between 4.3 to $8.7 \mathrm{Mb}$ ), McClure et al [29] identified QTLs for carcass weight in Angus cattle. The significance SNP (BTA-54082-nors) on BTA $22(32 \mathrm{Mb})$ contains FRMD4B gene. In mice, this gene plays a key role in actin cytoskeleton dynamics and membrane trafficking during junction remodeling and epithelial polarization [30]. QTL mapping by [31] detected QTL for carcass weight on BTA22 (28.5 to $45.7 \mathrm{Mb})$ in Bos indicus-Bos taurus cross. Interestingly, one of our significant SNP (BTB00874898) lies within the reported QTL region. The SNP on BTA12 (Hapmap51939-BTA-21630) is located within the PARP4 gene. This gene was known to have a key role in regulating adipogenesis [32]. McClure et al [29] mapped QTL regions associated with carcass weight on BTA 2 (62.2 to 75.6 $\mathrm{Mb}$ ), BTA 6 (45.9 to $53.1 \mathrm{Mb}$ ), 24 between 26.6 to $30.0 \mathrm{Mb}$ and BTA28 (4.3 to $8.7 \mathrm{Mb}$ ) in Angus cattle breed. Genome-wide association study identified QTL for carcass weight on BTA6 in Japanese Black cattle [9]. Similarly, several SNPs were highly
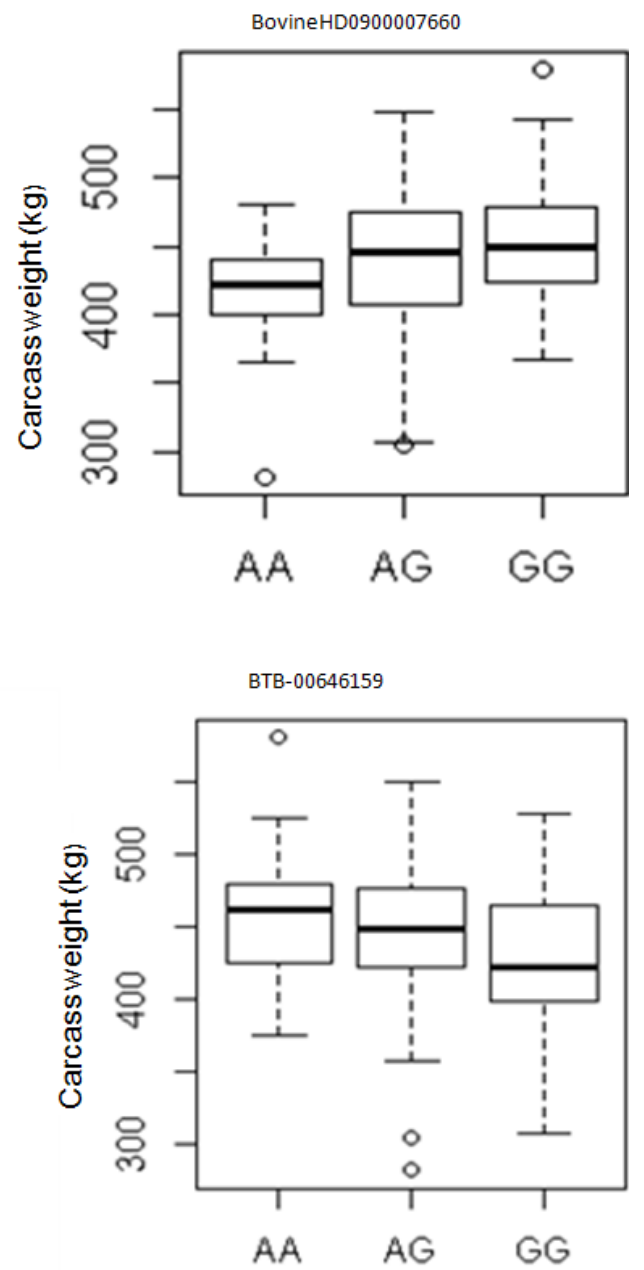

Figure 2. The effect of genotypes of four single nucleotide polymorphism identified to be associated with carcass weight in Hanwoo cattle for additive model. 
associated with growth on BTA6 between 25 and $53 \mathrm{Mb}$ on BTA 6 [33]. Cattle QTL for birth weight [34,35], pre- and postweaning body weight gain [35,36], and yearling weight [34] were reported from 25 to $53 \mathrm{Mb}$ on BTA6.

Haplotype-based association analyses have been a powerful approach to detecting the causative variants underlying complex phenotypes $[21,37]$. To examine whether any haplotype was associated with carcass weight, we also performed haplotype regression analysis. Twelve haplotype blocks distributed in 9 chromosomes were shown to have a significant $(\mathrm{p}<0.05)$ effect on carcass weight. A highly significant $(p=0.0002)$ effect on carcass weight was found for haplotypes detected on BTA12. This haplotype block spanned $119.855 \mathrm{~kb}$ and contains two SNPs (Hapmap51939-BTA-21630 and ARS-BFGL-NGS-32801) within PARP4 and ring finger protein 17 (RNF17) genes. In GWAS, it has been demonstrated that RNF17 gene was associated with the fatty acid profile in Nellore cattle [38] and growth traits [39]. We found that a haplotype block of $3.591 \mathrm{~kb}$ (SNPs BovineHD1900016031 and BovineHD1900016032) on BTA19 had also a significant association with carcass weight $(p=0.001)$ and harbors transmembrane protein 94. Another region associated with carcass weight $(\mathrm{p}=0.02)$ was located on BTA20 encompassing a single annotated gene, ADAM metallopeptidase with thrombospondin type 1 motif $12(A D$ -
AMTS12). Recent co-association network analysis revealed that PR domain containing 16 (PRDM16) was among the key transcription factors regulating growth traits in pigs [39]. This gene is also involved in the differentiation of adipose tissue [40]. Interestingly, ADAMTS12 identified as co-associated with PRDM16 [39]. In human, ADAMTS12 is involved in skeletal muscle regeneration and mediates the neurotoxic effect of beta-amyloid peptide [41].

The $M y o D$ gene family encodes basic helix-loop-helix proteins and are involved in muscle cell determination and differentiation [42,43]. Interestingly, our gene-network analysis uncovered key transcription factors associated with myogenesis including myogenin and lymphoid enhancer-binding factor 1 transcription factors related genes (Figure 3). A study in beef cattle has shown that myogenin (MYOG) was up-regulated in cells from Wagyu $\mathrm{x}$ when compared to cells from Angus and Hereford [44]. In another study, it has been found that variation in myogenic factor 5 (MYF5), myogenic factor 6 (MYF6) and $M Y O G$ genes were associated with growth and carcass traits in pigs [45]. LEF-1 transcription factor is an element of canonical Wnt pathway, which is required to induce myogenesis [46]. In pigs, variation in MYOG has been associated with increased meat production [47].

We did not detect common SNPs with previous studies

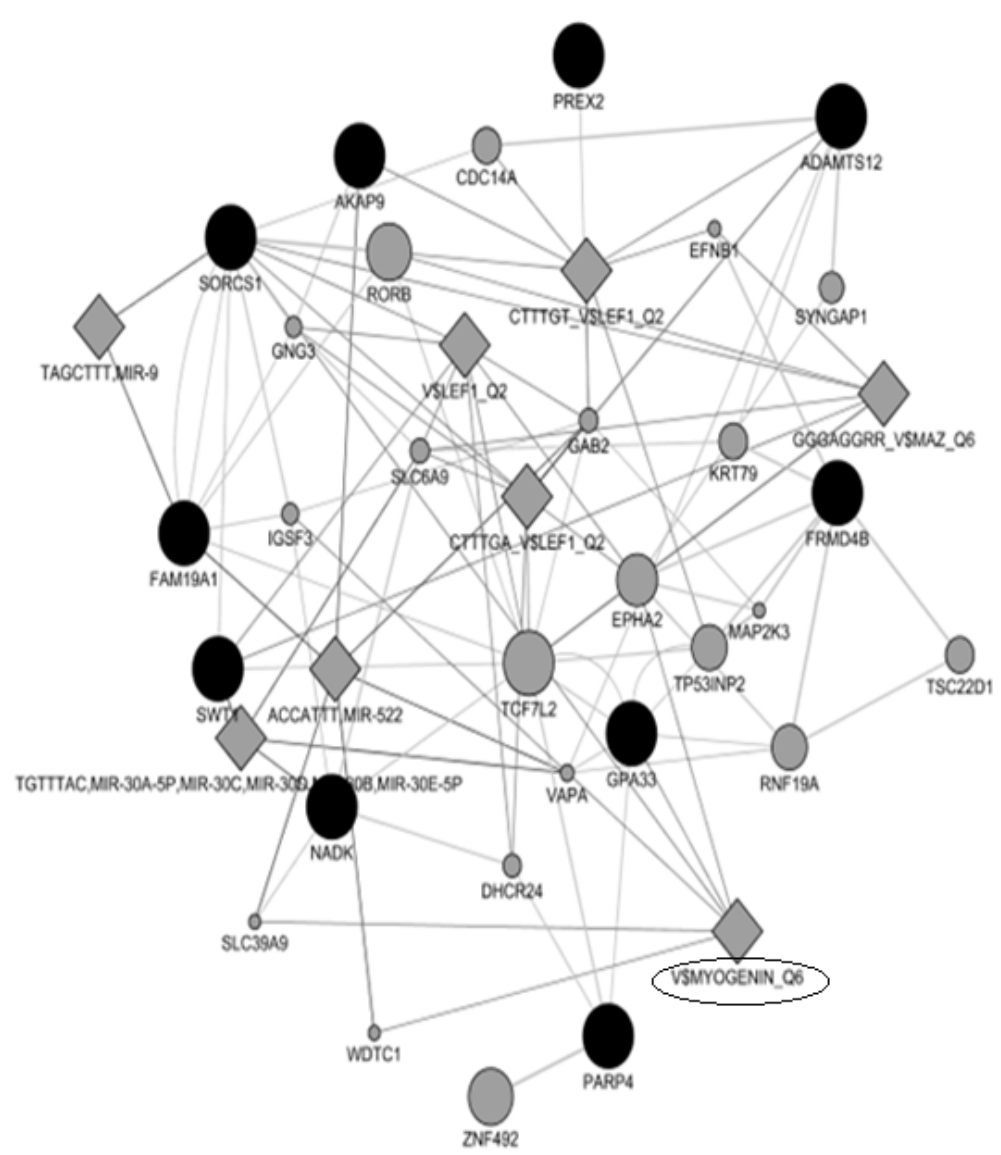

Figure 3. Results of gene networks analysis. Dark and gray circles represent candidate genes and associated genes, respectively. 
carried out in Hanwoo cattle $[11,24]$. The lack of overlap with previous studies might be attributed to the difference in SNP density, population sample size and, and lack of detection power $[48,49]$. Additionally, in the present study, we used carcass weight of commercial Hanwoo cattle measured at an average age of 31 months, whereas in the previous GWAS, Hanwoo steers of 24 months of age from the progeny-testing program were analyzed [25]. The association of distinct QTL with body weight and growth rates at different developmental stages has been well demonstrated in model and farm animal species $[17,18]$. The difference in age between our study population and previous studies might have affected to detect common significant loci for carcass weight. Different genes and physiological pathways may contribute to an observed difference in body weight /carcass weight at various developmental stages. Therefore, it will be yet possible to investigate QTLs for carcass weight at different ages and physiological phases in the Hanwoo cattle.

The lack of detection of SNPs that reached the genome-wide significant level is seemingly due to small sample size used in our study. A sufficiently large sample size is required to detect variants that account for a moderate proportion of genetic variance [7]. Therefore, validation of the most significant SNPs using reasonable sample size is required to design SNP-assisted selection program in commercial Hanwoo cattle. Yet, some of the SNPs identified in this study may help to understand the molecular mechanisms that determine beef production in Hanwoo cattle.

\section{CONFLICT OF INTEREST}

We certify that there is no conflict of interest with any financial organization regarding the material discussed in the manuscript. Ku J, Seo S are employees of Turbosoft Inc..

\section{ACKNOWLEDGMENTS}

This research was supported by the grants of Chungbuk Industry University Cooperation Institute in 2016 and "Leaders in Industry-university Cooperation +" project by the Ministry of Education and National Research Foundation of Korea.

\section{REFERENCES}

1. Mannen H, Kohno M, Nagata Y, et al. Independent mitochondrial origin and historical genetic differentiation in North Eastern Asian cattle. Mol Phylogenet Evol 2004;32:539-44.

2. Lee S-H, Park B-H, Sharma A. Hanwoo cattle: origin, domestication, breeding strategies and genomic selection. J Anim Sci Technol 2014;56:2.

3. Park B, Choi T, Kim S, Oh S-H. National genetic evaluation (system) of Hanwoo (Korean native cattle). Asian-Australas
J Anim Sci 2013;26:151-6.

4. Irshad A, Kandeepan G, Kumar S, et al. Factors influencing carcass composition of livestock: A review. J Anim Prod Adv 2013; 3:177-186.

5. Wythes J, Shorthose W, Dodt R, Dickinson R. Carcass and meat quality of Bos indicus $\times$ Bos taurus and Bos taurus cattle in northern Australia. Aust J Exp Agric 1989;29:757-63.

6. Zhang H, Wang Z, Wang S, Li H. Progress of genome wide association study in domestic animals. J Anm Sci Biotechnol 2012;3:26.

7. Stranger BE, Stahl EA, Raj T. Progress and promise of genomewide association studies for human complex trait genetics. Genetics 2011;187:367-83.

8. Cericola F, Portis E, Lanteri S, et al. Linkage disequilibrium and genome-wide association analysis for anthocyanin pigmentation and fruit color in eggplant. BMC Genomics 2014; $15: 896$.

9. Nishimura S, Watanabe T, Mizoshita K, et al. Genome-wide association study identified three major QTL for carcass weight including the PLAG1-CHCHD7 QTN for stature in Japanese Black cattle. BMC Genet 2012;13:40.

10.Setoguchi K, Furuta M, Hirano T, et al. Cross-breed comparisons identified a critical 591-kb region for bovine carcass weight QTL (CW-2) on chromosome 6 and the Ile-442-Met substitution in NCAPG as a positional candidate. BMC Genet 2009; 10:43.

11.Lee SH, Choi BH, Lim D, et al. Genome-wide association study identifies major loci for carcass weight on BTA14 in Hanwoo (Korean cattle). PLOS ONE 2013;8:e74677.

12. Lee SH, van der Werf J, Lee SH, et al. Genome wide QTL mapping to identify candidate genes for carcass traits in Hanwoo (Korean Cattle). Genes Genom 2012;34:43-9.

13. Nishimura S, Watanabe T, Mizoshita K, et al. Genome-wide association study identified three major QTL for carcass weight including the PLAG1-CHCHD7 QTN for stature in Japanese Black cattle. BMC Genet 2012;13:40.

14. Takasuga A, Watanabe T, Mizoguchi Y, et al. Identification of bovine QTL for growth and carcass traits in Japanese Black cattle by replication and identical-by-descent mapping. Mamm Genome 2007;18:125-36.

15. Sorbolini S, Bongiorni S, Cellesi M, et al. Genome wide association study on beef production traits in Marchigiana cattle breed. J Anim Breed Genet 2017;134:43-8.

16. Jiang Z, Michal JJ, Chen J, et al. Discovery of novel genetic networks associated with 19 economically important traits in beef cattle. Int J Biol Sci 2009;5:528-42.

17. Cheverud JM, Routman EJ, Duarte FAM, et al. Quantitative trait loci for murine growth. Genetics 1996;142:1305-19.

18. Podisi BK, Knott SA, Burt DW, Hocking PM. Comparative analysis of quantitative trait loci for body weight, growth rate and growth curve parameters from 3 to 72 weeks of age in female chickens of a broiler-layer cross. BMC Genet 2013; 
$14: 22$.

19. Middelbos IS, Vester BM, Karr-Lilienthal LK, Schook LB, Swanson KS. Age and diet affect gene expression profile in canine skeletal muscle. PLOS ONE 2009;4:e4481.

20. Kang HM, Sul JH, Service SK, et al. Variance component model to account for sample structure in genome-wide association studies. Nat Genet 2010;42:348-54.

21.Gabriel SB, Schaffner SF, Nguyen H, et al. The structure of haplotype blocks in the human genome. Science 2002;296: 2225-9.

22. Fortes MR, Reverter A, Nagaraj SH, et al. A single nucleotide polymorphism-derived regulatory gene network underlying puberty in 2 tropical breeds of beef cattle. J Anm Sci 2011;89: 1669-83.

23. Shannon P, Markiel A, Ozier O, et al. Cytoscape: a software environment for integrated models of biomolecular interaction networks. Genome Res 2003;13:2498-504.

24. Kim Y, Ryu J, Woo J, et al. Genome-wide association study reveals five nucleotide sequence variants for carcass traits in beef cattle. Anim Genet 2011;42:361-5.

25. Lee SH, Choi BH, Lim D, et al. Genome-wide association study identifies major loci for carcass weight on BTA14 in Hanwoo (Korean cattle). PLOS ONE 2013;8:e74677.

26. Espigolan R, Baldi F, Boligon AA, et al. Associations between single nucleotide polymorphisms and carcass traits in Nellore cattle using high-density panels. Genet Mol Res 2015;14:1113344.

27. Rempel LA, Casas E, Shackelford SD, Wheeler TL. Relationship of polymorphisms within metabolic genes and carcass traits in crossbred beef cattle. J Anim Sci 2012;90:1311-6.

28. Utsunomiya YT, do Carmo AS, Carvalheiro R, et al. Genomewide association study for birth weight in Nellore cattle points to previously described orthologous genes affecting human and bovine height. BMC Genet 2013;14:52.

29. McClure MC, Morsci NS, Schnabel RD, et al. A genome scan for quantitative trait loci influencing carcass, post-natal growth and reproductive traits in commercial Angus cattle. Anim Genet 2010;41:597-607.

30.Ikenouchi J, Umeda M. FRMD4A regulates epithelial polarity by connecting Arf6 activation with the PAR complex. Proc Natl Acad Sci USA 2010;107:748-53.

31. Kim JJ, Farnir F, Savell J, Taylor JF. Detection of quantitative trait loci for growth and beef carcass fatness traits in a cross between Bos taurus (Angus) and Bos indicus (Brahman) cattle. J Anim Sci 2003;8:1933-42.

32. Rouleau M, Patel A, Hendzel MJ, et al. PARP inhibition: PARP1 and beyond. Nat Rev Cancer 2010;10:293-301.

33. Snelling WM, Allan MF, Keele JW, et al. Genome-wide association study of growth in crossbred beef cattle. J Anim Sci 2010;88:837-48.
34. Casas E, Shackelford SD, Keele JW, et al. Quantitative trait loci affecting growth and carcass composition of cattle segregating alternate forms of myostatin. J Anim Sci 2000;78:560-9.

35. Kneeland J, Li C, Basarab J, Snelling W, et al. Identification and fine mapping of quantitative trait loci for growth traits on bovine chromosomes 2, 6, 14, 19,21, and 23 within one commercial line of Bos taurus. J Anim Sci 2004;82:3405-14.

36. Kim JW, Park SI, Yeo JS. Linkage mapping and QTL on chromosome 6 in Hanwoo (Korean cattle). Asian-Australas J Anim Sci 2003;16:1402-5.

37. Daly MJ, Rioux JD, Schaffner SF, Hudson TJ, Lander ES. Highresolution haplotype structure in the human genome. Nat Genet 2001;29:229-32.

38.Lemos MVA, Chiaia HLJ, Berton MP, et al. Genome-wide association between single nucleotide polymorphisms with beef fatty acid profile in Nellore cattle using the single step procedure. BMC Genomics 2016;17:213.

39. Puig-Oliveras A, Ballester M, Corominas J, et al. A co-association network analysis of the genetic determination of pig conformation, growth and fatness. PLOS ONE 2014;9:e114862.

40. Seale P, Bjork B, Yang W, Kajimura S, et al. PRDM16 controls a brown fat/skeletal muscle switch. Nature 2008;454:961-7.

41. Laumet G, Petitprez V, Sillaire A, et al. A study of the association between the ADAM12 and SH3PXD2A (SH3MD1) genes and Alzheimer's disease. Neurosci Lett 2010;468:1-2.

42. Olson EN. MyoD family: a paradigm for development? Genes Dev 1990;4:1454-61.

43. Weintraub H, Davis R, Tapscott S, et al. The MyoD gene family: Nodal point during specification of the muscle cell lineage. Science 1991;251:761-6.

44. Coles CA, Wadeson J, Leyton CP, et al. Proliferation rates of bovine primary muscle cells relate to liveweight and carcase weight in cattle. PLOS ONE 2015;10:e0124468.

45. Cieslak D, Kuryl J, Kapelanski W, et al. Relationship between genotypes at at MYOG, MYF3 and MYF5 loci and carcass meat and fat deposition traits in pigs. Animal Science Papers and Reports (Poland); 2002.

46. Brunelli S, Relaix F, Baesso S, Buckingham M, Cossu G. Beta catenin-independent activation of MyoD in presomitic mesoderm requires $\mathrm{PKC}$ and depends on Pax3 transcriptional activity. Dev Biol 2007;304:604-14.

47. Te Pas M, Harders F, Soumillion A, et al. Genetic variation at the porcine MYF-5 gene locus. Lack of association with meat production traits. Mamm Genome 1999;10:123-7.

48. Stahl EA, Raychaudhuri S, Remmers EF, et al. Genome-wide association study meta-analysis identifies seven new rheumatoid arthritis risk loci. Nat Genet 2010;42:508-14.

49. Clayton DG, Walker NM, Smyth DJ, et al. Population structure, differential bias and genomic control in a large-scale, casecontrol association study. Nat Genet 2005;37:1243-6. 